\title{
THE DETERMINATION OF ALVEOLAR CARBON DIOXIDE
}

\author{
H AROLD V. RICE, M.D., PH.D. ${ }^{*}$
}

THE determination of alveolar $\mathrm{CO}_{2}$ concentration depends on the measurement of the amount of $\mathrm{CO}_{2}$ absorbed from a $20 \mathrm{ml}$. sample of alveolar aur by a concentrated solution of $\mathrm{NaOH}$. Pressures are equilibrated to atmospheric at the start and finısh to elimmate false volume changes due to pressure differences. Temperature changes are insignificant because the procedure takes only a minute or less, and the apparatus does not change in temperature provided certain simple precautions are taken

\section{Apparatus}

This consists of a glass analysıs chamber which can be easily assembled (see diagram). The lower stem (a) consists of a section of a $5 \mathrm{ml}$ pipette, to measure $3 \mathrm{ml}$ graduated at $1 / 10 \mathrm{ml}$. The zero mark should be a $\mathrm{cm}$. or more from the lower end This $3 \mathrm{ml}$. pipette is connected by rubber tubing $(b)$ to a larger glass section $(c)$ consisting of giass tubing of approximately $1 \mathrm{~cm}$. inside diameter. A 2-inch length of rubber tubing (d) of relatively small bore (e.g., $5 \mathrm{~mm}$.) is attached to the upper end and clamped in the middle $(e)$. Both ends of the larger glass section are drawn out somewhat to permit connections to the rubber tubing without a shoulder The section of larger glass is cut to such a length that the internal capacity of the whole chamber from the zero mark to the clamp is $20 \mathrm{ml}, 1$ e., the larger section is of about $17 \mathrm{ml}$. capacity. This can be determined by filling the chamber from a $20 \mathrm{ml}$. syringe or burette, and adjustıng to final volume by sliding the glass tube the proper distance into the rubber connector (b).

The chamber is washed with water to wet the inside and mounted vertically on a stand with a burette clamp with the lower end immersed in water at room temperature so that the inside meniscus is exactly at the zero mark. The immersion reservoir $(f)$ should be deep enough to accept the total length of the $3 \mathrm{ml}$ pipette. (A tall beaker or short graduate is suitable.) The assembly is allowed to stand for a few-minutes before use to allow dranage of excess water from the inside, and to allow the glass to attain room temperature

\section{Procedure}

The sample of alveolar air is collected in a small anaesthetic bag ( $g$ ) This is attached to the upper end of the gas chamber by a glass connection. The bag is unclamped at the connector, the clamp $(e)$ on the gas chamber is squeezed open though not removed At the same time the sampling bag is squeezed so that the analysis chamber is flushed through several times and completely filled with the gas sample. The excess bubbles out of the lower end. When filled with the sample, clamp (e) is allowed to close, thus trapping the gas sample in the chamber with the meniscus at or near the tip of the measuring pipette and below the open surface of the water in the reservor.

"Department of Physiology and Pharmacology, University of Alberta 
Owing to the handling of the sampling bag the entrapped sample will be warmer than room temperature, and will immediately start to cool and shrink, causing the meniscus to rise in the pipette. The meniscus will however become stable at a level below the zero mark as soon as the gas sample attains the same

FIGURE 1

\section{Diagram of $\mathrm{CO}_{2}$ Analyser}

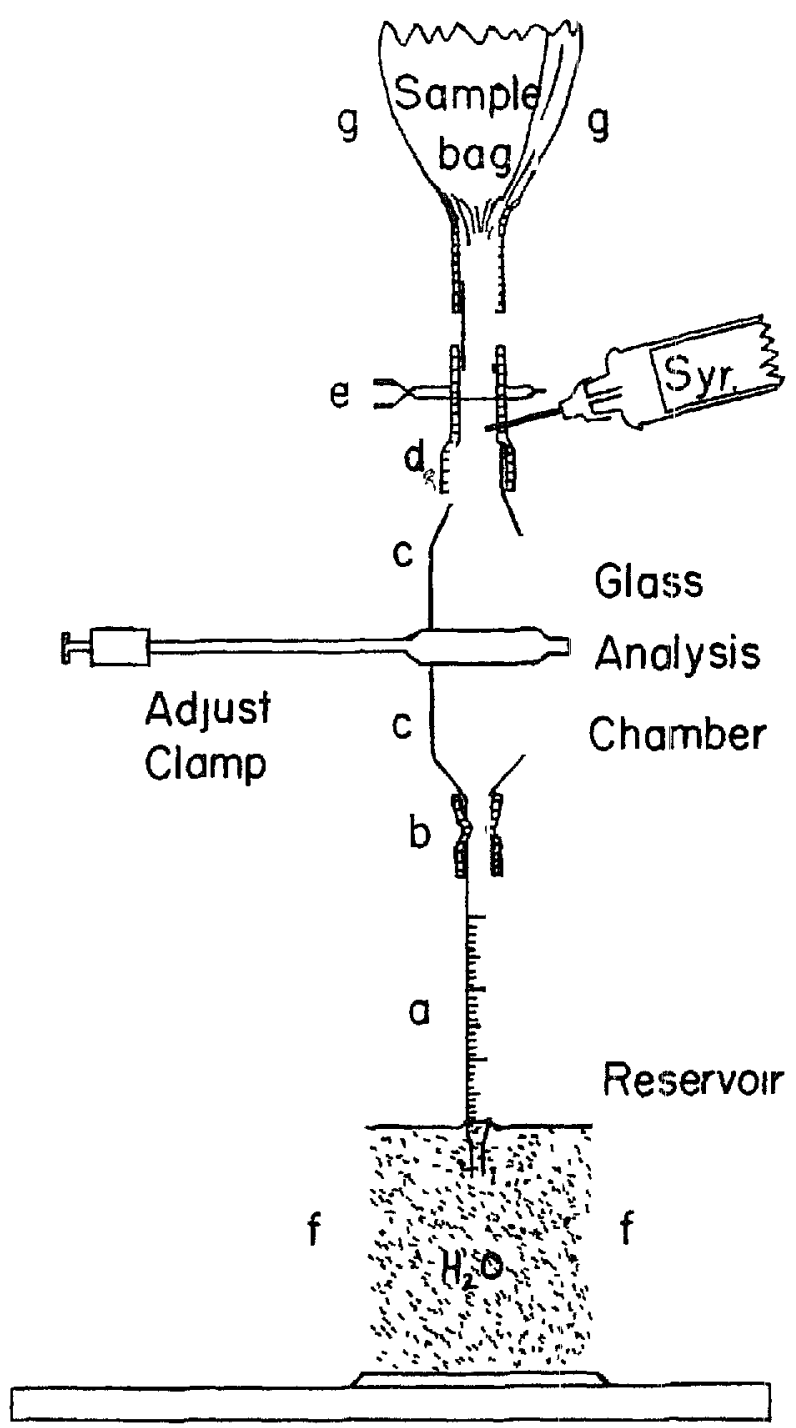

temperature as the glass chamber (which is room temperature).

Using a dry empty syringe and 25 gauge needle, gas is slowly withdrawn from the chamber by puncturing the rubber tubing $(d)$ below clamp $(e)$, until the inside meniscus is again at the zero mark on the pipette.

The analysis chamber thus contains exactly $20 \mathrm{ml}$. of gas sample at barometric pressure and room temperature.

Using a small syringe and 25 gauge needle, approximately $1 / 2 \mathrm{ml}$. (an excess) of a half-saturated solution of $\mathrm{NaOH}$ is slowly injected into the chamber through the rubber tubing $(d)$. The needle is directed so as to allow the $\mathrm{NaOH}$ to flow down the inside wall of the chamber on opposite sides thus providing a large absorption surface. The excess trickles down into the pipette and collects at the 
water surface. Care must be taken to inject the $\mathrm{NaOH}$ slowly to avoid an accumulation of it at the rubber connector $(b)$ which might occlude the smaller bore of the pipette. The contained $\mathrm{CO}_{2}$ will immediately start to be absorbed and the meniscus will rise in the pipette. When absorption is complete $\mathrm{i}$ : will attan a constant level. The chamber is then lowered on the stand unt 1 the menusc inside the pipette and outside in the water tank are at the same level. The entrapped gas residue after $\mathrm{CO}_{2}$ absorption is again at baromtric pressure and at room temperature.

The amount of $\mathrm{CO}_{2}$ absorbed is the volume of the water now occupying the pipette from the zero mark (startung level) to the final reading of the meniscus. As the original alveolar sample was $20 \mathrm{ml}$., the per cent composition of $\mathrm{CO}_{2}$ is obtamed by multiplying; the amount actually absorbed by 5 . Example: amount actually absorbed, $1.1 \mathrm{ml}$; per cent $\mathrm{CO}_{2}$ composition of alveolar gas sample is 5.5 .

The apparatus is thoroughly washed through with water following each test and again mounted in position for the next analysis. This allows time for the excess water to drain off, and for the glass to return to room temperature.

\section{Precautions}

The most serious potential errors are changes in volume which would occur if the temperature of the gas changed during the test. Such temperature changes are readily avoided if the glass is at room temperature throughout the test. Since the procedure is brief, there is insufficient time for fluctuations of room temperature to affect the temperature of the glass significantly. However, errors will occur if the glass was warmer or cooler than the room prior to the start of the test, and progressively cooled or warmed as it approached room temperature during the test Progressive expansion of the gas due to warming of the chamber while absorption with $\mathrm{NaOH}$ was proceeding would offset the absorption and give low readings, progressive contraction of the gas due to cooling of the chamber would reduce the total gas volume and thus add to the shrinkage due to absorption of $\mathrm{CO}_{2}$, and give high readings.

Therefore, the chamber should not be moved from one position to another (e g., from a sunny or cold window, or from a position near a radiator to a more remote position) unless time is allowed for the glass to attain the air temperature of the new position. Similarly, the glass should not be handled immediately prior to, or during, a test, or washed with hot or cold water.

To avord these possible errors the apparatus should preferably be used in a sheltered place in the room against an inside wall, and washed from a tank of water which has been standing for some time, and is, therefore, already at room temperature. The charaber should also be handled at the burette clamp rather than by grasping the g-ass in the hand.

Depending on the fineness of the $1 / 10 \mathrm{ml}$. graduations of the pipette, readings can be made accurate y to one-half division or better, i.e., $0.05 \mathrm{ml}$., giving an accuracy of approximately 0.25 per cent $\mathrm{CO}_{2}$ concentration. This is adequate for most clinical work, and is probably less than the sampling error. Concentrations of $\mathrm{CO}_{2}$ up to 15 per cent can be determined.

This technique is easily mastered, and is suitable for $\mathrm{CO}_{2}$ determinations on 
a hospital ward. The apparatus can be assembled cheaply from sưplies avalable in standard laboratories. Its chef merit is the rapidity with whic $\mathrm{CO}_{2}$ determinations can be made."

\section{The Collection of Alveolar Air Samples fior $\mathrm{CO}_{2}$ Analysis}

The greatest source of error in attempting to determine the alveolar $\mathrm{CO}_{2}$ concentration lies $\mathrm{m}(a)$ the difficulty of obtaining true alveolar air samples; (b) the possibility that, because the respiratory rhythm may be altered when the subject gives the sample, $\mathrm{CO}_{2}$ may accumulate in, or be blown off from, the alveoli just prior to the sampling. The alveolar $\mathrm{CO}_{2}$ concentration would thus not be the proper value for the patient at that time.

The first possible sampling error requires that care be taken to collect an expired sample only after the air in the dead space is expelled. Since the dead space gas amounts to approxumately $130 \mathrm{ml}$. and is low in $\mathrm{CO}_{2}$, contamination of the sample by gas collected early in expiration will cause low readings. Only the latter $1 / 2$ or $1 / 3$ of the expred volume should be collected.

If the patient has a tracheotomy and is in a respirator this is easy. By watching the neck seal of the respirator (or some other part that moves in accordance with the pressure changes), one can easily learn to make quick contact between the nipple of the sampling bag and the tracheotomy tube at approximately midpoint of expiration. By opening a spring clip on the bag at the same time, samples can be collected from a series of expirations until the bag is filled.

If the patient has not got a tracheotomy the technique is more difficult and one has to obtain co-operation through practice, using a mouthpiece (or mask) Alternatively, a Haldane tube, or similar divice can be used

It should always be remembered that a rubber anaesthetic bag, even when empty and collapsed, usually contains considerable gas either from the atmosphere or from a previous sample. This residue will dilute the first sample taken from the next subject.

Therefore, the bag should first be filled with a prelimmary sample from the subject, and then emptied. This will wash out almost all the original residue of gas in the bag. A second collection from the patient will then be undiluted by gas from extraneous sources.

Recognizing its potential errors, the author much prefers bag sampling of alveolar aur obtained by accumulating the tidal end-displacement of several expirations, rather than analysis of a single end-sample. The flexibility of the bag is also preferred to rigid devices, such as a syringe or pump.

When the sample is to be obtamed from patients breathing on their own the technique of collecting end-samples without changing the respiratory rhythm can usually only be developed with practice. Some time spent on this with the patient will usually result in a significant improvement in the constancy of results. The greatest variation occurs in patients who try too hard to help. and are not relaxed, or who are too ill to co-operate *

"An alternative method for free $\mathrm{CO}_{2}$ analysis has also been developed which is "dry", and employs no solutions It is portable, even quicker to carry out than the above method, and accurate to 01 per cent composition of $\mathrm{CO}_{2}$ in the sample Arrangements for manufacture of the apparatus are being, made

* The problem of samp ing errors is considered to be so important that much tme has been 


\title{
LA DETERMINATION DU DIOXIDE DE CARBONE ALVEOLAIRE
}

\author{
Harold V. RiCe, M.D., PH D.
}

LA détermination de la concentration du $\mathrm{CO}_{2}$ alvéolarre dépend de la mesure de la quantité de $\mathrm{CO}_{2}$ absorbé par une solution concentrée ce $\mathrm{NaOH}$ à partir d'un échantillon de $20 \mathrm{cc}$. d'air alvéolaire. Les pressions son'équilibrées à la pression athmosphérique au début et à la fin pour éliminer les changements erronés de volumes dus aux différences de pression. Les changements de température étant minımes, l'opération prend au maximum une minute et la température de l'apparell ne change pas, pourvu que certaines précautions élémentarres soient prises.

\section{ApPareIL}

Il consiste en une chambre d'analyse en verre qui s'assemble aisément (voir diagramme). La tige inférieure (a) se compose d'une section de pipette de 5 cc. calculée pour mesurer $3 \mathrm{cc}$. et graduée au $1 / 10$ de $\mathrm{m}$. Le point zéro doit se trouver à un centımètre ou plus de l'extrémité inférieure. Cette pipette de $3 \mathrm{cc}$. est reliée par un tube de caoutchouc $(b)$ à une section de verre plus large (c) consistant en un tube de verre d'environ $1 \mathrm{~cm}$. de diamètre intérieur. Un tube de caoutchouc long de deux pouces $(d)$ et d'un dramètre intérieur relativement petit (par exemple $5 \mathrm{~mm}$.) est attaché à la partie supérieure et pris dans une pince en son milier $(e)$. Les deux extrémités de la section de verre la plus large sont étrées quelque peu de mamère à permettre les raccords sans soutien avec les tubes de caoutchouc. La section de verre la plus large est coupée à une longueur telle que la capacité intérieure de l'ensemble de la chambre à partir du point zéro jusqu'à la pınce soit de $20 \mathrm{ml}$., c'est à dire que la capacité de la section la plus large soit d'environ $17 \mathrm{ml}$. On peut déterminer cela en remplissant la chambre à partur d'une seringue ou burette de $20 \mathrm{ml}$., en réglant le volume final et en fassant ghsser le tube de verre dans le raccord de.caoutchouc.

On lave la chambre avec de l'eau pour mouiller l'intérieur et on la monte vertıcalement sur un support à burettes en immergeant l'extrémité inférieure dans de l'eau à la température environnante de manière à ce que le ménisque intérneur solt exactement, au point zéro. Le réservoir d'immersion $(f)$ doit être assez profond pour recevoir la longueur totale de la pipette de $3 \mathrm{ml}$. (Un grand bécher ou un petıt verre gradué convienne.) On attendra quelques minutes avant d'employer l'appareil pour permettre le drannage de l'eau en surplus à l'intérieur et pour permettre au verre d'atteindre la température environnante.

spent in developing a device through which the satient simply breathes and which automatically rejects the first portion of the expired vo ume, and colects. the latter portion The amount rejected can be adjusted to the size of the patient The patient is not required to control his respiratory movements in any manner during the sampling He is required only to breathe casually and naturally through the apparatus, which is so designed as to provide minimal resistance, dead-space, etc.

While relatively simple, this automatic alveolar air sampler requires some machining in its construction Its assembly is, therefore, not practical in a routine laboratory. Investigations into the possibility of having it manufactured are now in progress. 


\section{OpÉration}

L'échantillon d'air alvéolaire est receuilli dans un petit ballon à anesthésie (g). Celui-ci est attaché à. l'extrémité supérieure de la chambre à gaz par un raccord de verre. On relâche la pince du ballon au niveau du raccord et la pince (e) de la chambre à gaz est ouverte, mais non retirée. Au même instant, le ballon échantillion est comprimé de telle sorte que la chambre d'analyse soit purgée à plusieurs reprises et complètement remplie du gaz échıntillon. L'excès bar jotte à la sortie inférieure. Lorsque le plein est achevé, la pince (e) est refermée, emprisonnant ainsi l'échantillon de gaz dans la chambre, avec le ménisque au niveau de ou près de la pointe de la pipette à mesurer et au dessous de la surface libre de l'eau du réservoir.

Par suite de la manipulation du ballon échantillon, l'échantillon emprisonné sera plus chaud que la température environnante, et commencera immédiatement à refroidir et à se contracter, causant une élévation du ménisque dans la pipette. Le ménisque se stabilisera cependant au dessous du point zéro aussitôt que l'échantillon de gaz atteindra la même température que la chambre de verre (qui est la température environnante).

A l'aide d'une seringue vide et sèche et d'une aiguille de calibre 25 , on retrre lentement le gaz en ponctionnant le tube de caoutchouc $(d)$ au dessous de la pince $(e)$, jusqu'à ce que le ménısque intérieur atteigne à nouveau le point zéro sur la pipette.

La chambre d'analyse contient ainsı exactement $20 \mathrm{cc}$. d'échantllon de gaz à la pression barométrique et à la température environnant.

A l'aide d'une petite seringue et d'une aiguille de calbre 25 , on injecte lentement dans la chambre au travers du tube de caoutchouc $(d)$ environ $1 / 2 \mathrm{cc}$. (excès) d'une solution de $\mathrm{NaOH}$ à demi saturée. On dırige l'aigulle de manière à permettre au $\mathrm{NaOH}$ de couler le long de la paroi intérieure de la chambre sur les deux côtés opposés, offrant ainsı une grande surface d'absorption. L'excès coule goutte à goutte dans la pipette et se ramasse à la surface de l'eau. Il faut veiller à injecter le $\mathrm{NaOH}$ doucement pour éviter qu'll ne s'accumule au niveau du raccord de caoutchouc $(b)$ et n'obstrue ainsi le plus petit diamètre intérieur de la pipette. Le $\mathrm{CO}_{2}$ qui se trouve à l'intérieur commencera immédiatement à être absorbé et le ménısque montera dans la pipette. Quand l'absorption sera complète, il atteindra un niveau constant. On abaisse ensuite la chambre sur le support jusqu'à ce que les ménisques à l'nntérieur de la pipette et à l'extérieur dans le réservoir à eau soient au même niveau Le résidu du gaz emprisonné, après absorption du $\mathrm{CO}_{2}$, est à nouveau à la pression barométrique et à la température environnante.

La quantité de $\mathrm{CO}_{2}$ absorbé est le volume de l'eau qui occupe maintenant la pipette depuis le point zéro (niveau de départ) jusqu’à la dernière lecture du ménisque. Comme l'échantillon alvéolaire original était: de $20 \mathrm{ml}$., la composition de $\mathrm{CO}_{2}$ (en pourcentage) s'obtient en multipliant par 5 la quantité effectivement absorbée. Exemple : quantité effectivement absorbée, 1,1 ml. ; teneur (pourcentage) en $\mathrm{CO}_{2}$ de l'échantillon de gaz alvéolaire : 5,5 .

Après chaque test, on lave soigneusement l'a jpareil avec de l'eau et on le remonte pour l'analyse suivante. Ceci permet à _'excès d'eau de s'écouler et au verre de revenir à la température environnante. 


\section{PhÉcautions}

Les erreurs possibles les plus sérieuses sont les changements de volume qui se produisent sı la température du gaz change pendant l'expérience. Ces changements de température 's'évitent tacilement si le verre reste à la température environnante pendant tout le test. L'opération étant courte, les fluctuations de la température environnante n'ont pas le temps d'affecter sensiblement la température du verre. Cependant des erreurs se produiront si le verre était plus chaud ou plus froid que la salle avant le début de l'expérience, et s'il s'est progressivement réchauffé ou refroidi jusqu'à approcher de la température environnante pendant l'expérience. Une dilatation progressive du gaz due à l'échauffement de la chambre pendant l'absorption par $\mathrm{NaOH}$ compenserait l'absorption et donnerait des lectures trop basses : une contraction progressive du gaz due au refroidissement de la chambre réduirait le volume total du gaz et accroîtrait ainsi la contraction due à l'absorptıon de $\mathrm{CO}_{2}$, et donnerait des lectures trop élevées.

Par conséquent, on ne doit pas déplacer la chambre (par exemple, d'une fenêtre ensolelllée ou froide, ou d'une position près d'un radiateur à une position plus éloignée) à moins qu'on ne laisse au verre le temps d'atteindre la température environnante dans sa nouvelle position. De même, le verre ne doit pas être manipulé juste avant, ou pendant, une expérience, ni lavé avec de l'eau chaude ou froide.

Pour éviter ces erreurs possibles, l'apparell sera employé de préférence dans un endroit abrité le la salle, contre une paroi intérieure, et lavé dans un réservoir d'eau qui aura été placé dans la salle quelque temps avparavant et se trouve donc déjà à la température environnante. La chambre devreit aussi être manipulée au niveau de la pince à burettes plutôt qu'en prenant le verre dans la main.

Suivant la finesse des graduations au $1 / 10$ de cc. de la pipette, on peut faire avec exactitude des lectures à une demi-division près c'est-à-dire $0.05 \mathrm{cc}$., ou plus précises, indiquant la concentration en $\mathrm{CO}_{2}$ avec une exactitude d'environ 0.25 pour cent. Ceci convient pour la plupart des travaux cliniques et reste sans doute au dessous de l'erreur d'échantillonage. Des concentrations en $\mathrm{CO}_{2}$ s'élevant jusqu'à 15 pour cent peuvent être déterminées.

Cette technique se possède facilement et convient aux déterminations de $\mathrm{CO}_{2}$ dans les salles d'hôpital. L'appareil peut être monté à peu de frais à partir d'éléments disponıbles dans les laboratoires standard. Son principal mérite est la rapidité avec laquelle on peut effectuer les déterminations en $\mathrm{CO}_{2}{ }^{*}$

\section{LA RÉCOLTE DES ÉCHANTILLONS D'AIR ALVÉOLAIRE POUR L'ANALYSE DE $\mathrm{CO}_{2}$}

La majeure source d'erreurs, quand on tente de déterminer la concentration du $\mathrm{CO}_{2}$ alvéolaire, réside dans $(a)$ la defficulté d'obtenir de véritables échantillons d'air alvéolaire; $(b)$ l'éventualité que, parce que le rythme respiratoire du sujet peut être altéré quand il donne l'échantillon, $\mathrm{CO}_{2}$ puisse s'accumuler dans, ou être expulsé des alvéoles juste avant l'échantillonage. La concentration du $\mathrm{CO}_{2}$ alvéolaire ne serait pas, dans ce cas, la teneur réelle pour le patient à ce moment.

\footnotetext{
- Une autre méthode pour l'analyse de $\mathrm{CO}_{2}$ Lbre a été également mise au point . cette méthoce est * sèche , et n'exige pas de solutions. Elle est portative, plus rapıde que la méthoc e ci-dessus, et plus précise jusqu’à une teneur en $\mathrm{CO}_{2}$ de 1 pour cent dans l'échantillon. Des arrangements pour la fabrication de l'appareil sont en cours
} 
La première possibulité d'erreur d'échantillonage exige qu'on veille à ne recueillir un échantillon qu'après éjectıon de l'air dans les espaces nuisıbles. Le gaz des espaces nuisibles s'élevant à environ $130 \mathrm{cc}$. et sa teneur en $\mathrm{CO}_{2}$ étant faible, la contamination de l'échantıllon par du gaz recúellli prématurément pendant l'expiration provoquera des lectures trop basses. La moitié ou le tiers seulement du volume expiré devrait être recueillı.

S1 le patient n'a pas de trachéotomie, la technique est plus difficile et il faut l'opération est assée. En regardant le collet du poumon d'acier au cou (ou toute autre partie qui se déplace suivant les variations de pression), on peut faclement' apprendre à établır un contact rapıde entre le mamelon du ballon-échantıllon et le tube à trachéotomie environ au milıeu de l'expıration. En ouvrant une pince à ressort sur le ballon an même instant, on peut recueillir des échantillons d'une série d'expirations jusqu’à remphssage du ballon.

Si le patient n'ax pas de trachéotomie, la technique est plus difficile et il faut obtenur la coopération du malade par la pratique et: l'emplo1 d'un tampon ou masque On peut également employer un tube de Haldane ou autre apparesl du même genre

On se souviendra toujours qu'un ballon à anesthésıe en caoutchouc, même s'il est vide et dégonflé, contient d'habitude une quantité considérable de gaz provenant soit de l'athmosphère soit d'un échantllon antérieur. Ce résidu diluera le premier échantillon pris sur le sujet suivant.

En conséquence, le ballon devra toujours être remplı d'un échantıllon prélımınaire du sujet, puis vidé Cette opération évacuera presque complètement le gaž résiduel contenu dans le ballon. Un deuxıème prélèvement effectué sur le patient ne sera pas alors dılué par des gaz d'orıgine étrangère.

Tout en admettant ses chances d'erreurs, l'auteur préfère de beaucoup l'échantıllonage de l'air alvéolaire dans un ballon, obtenu en accumulant l'air courant provenant de la dernière partie de plusieurs expırations, à l'analyse d'un unique échantillon de fin d'expiration

Il préfère aussı la flexıbilité du ballon aux dıspositifs rıgıdes, tels que seringues et pompes.

Quand il s'agit d'obtenir un échantillon sur des patients qui respirent d'euxmêmes, la technıque de prélever des échantlllons de fin de respiration sans changer le rythme respiratoure ne peut d'habitude être mise au point que par la pratique. Quelques instants passés avec le malade dans ce but se traduiront d'habitude par une nette amélioration dans la constance des résultats. Les variations les plus importantes se produsent chez les malades qui font trop d'efforts pour aider, et ne sont pas détendus, ou qui sont trop malades pour apporter leur concours."

${ }^{*}$ Le problème des erreurs d'échantillonage est consıdéré comme si important qu'on a passé beaucoup de temps à mettre au point un dispositif dans lequel le malade se borne à souffler ce qui rejette automatıquement la première partıe du volume expıré, et recueille la dernière portion. La quantité rejetée peut être réglée suivant la taille cu malade La malade n'est jas obligé de maitriser ses mouvements respiratorres en aucune façon pendant l'échantıllonage On lui demande seulement de respirer normalement et naturellement à travers l'appareil qui est conçu de manière à offrr une résistance, et un espace nussıble, moindre.

Quoique relativement simple, cet échantilloneur automatıque d'air alvéolaire demande quelque usinage dans sa construction. Son montage n'est donc pas pratique dans un laboratoire ordınaire. Une enquête quant à la possibilté de le faire fabriquer est en cours. 